\title{
Dual role of allele-specific DNA hypermethylation within the TERT promoter in cancer
}

\author{
Donghyun D. Lee, ,1,2,3 Martin Komosa, ${ }^{1,2}$ Sumedha Sudhaman, ${ }^{1,2}$ Ricardo Leão, ${ }^{2,4}$ Cindy H. Zhang, ${ }^{1,2}$ Joana D. Apolonio, ${ }^{1,2}$ \\ Thomas Hermanns, ${ }^{5}$ Peter J. Wild,,${ }^{6,7}$ Helmut Klocker, ${ }^{8}$ Farshad Nassiri, ${ }^{9}$ Gelareh Zadeh, ${ }^{9}$ Bill H. Diplas, ${ }^{10}$ Hai Yan, ${ }^{10}$ \\ Steven Gallinger, ${ }^{11}$ Trevor J. Pugh, ${ }^{12}$ Vijay Ramaswamy, ${ }^{2}$ Michael D. Taylor, ${ }^{2}$ Pedro Castelo-Branco, ${ }^{13,14,15,16}$ Nuno Miguel Nunes, ${ }^{1,2}$ \\ and Uri Tabori, ${ }^{1,2}$ \\ 'Program in Genetics and Genome Biology and ${ }^{2}$ The Arthur and Sonia Labatt Brain Tumour Research Centre, The Hospital for Sick Children, Toronto, Ontario, Canada. ${ }^{3}$ nstitute of Medical Science, Faculty \\ of Medicine, University of Toronto, Toronto, Ontario, Canada. ${ }^{4}$ Faculty of Medicine, University of Coimbra, Coimbra, Portugal. ${ }^{5}$ Department of Urology, University Hospital Zürich, University of Zurich, \\ Zurich, Switzerland. ${ }^{6} \mathrm{Dr}$. Senckenberg Institute of Pathology, University Hospital Frankfurt, Germany. ${ }^{7}$ Frankfurt Institute for Advanced Studies (FIAS), Frankfurt, Germany. ${ }^{8}$ Department of Urology, Medical \\ University of Innsbruck, Innsbruck, Austria. ${ }^{~}$ Division of Neurosurgery, University of Toronto, Toronto Western Hospital, University Health Network, Toronto, Ontario, Canada. ${ }^{10} \mathrm{Department}$ of Pathology, Duke \\ University Medical Center, Durham, North Carolina, USA. "Lunenfeld Tanenbaum Research Institute, Mount Sinai Hospital, University of Toronto, Toronto, Ontario, Canada. ${ }^{2}$ Princess Margaret Cancer Centre, \\ University Health Network, Toronto, Ontario, Canada. ${ }^{13}$ Faculdade de Medicina e Ciências Biomédicas (FMCB), Universidade do Algarve, Faro, Portugal. ${ }^{14}$ Algarve Biomedical Center Research Institute, Faro, \\ Portugal. ${ }^{15}$ Centre for Biomedical Research, University of Algarve, Faro, Portugal. ${ }^{16}$ Champalimaud Research Program, Champalimaud Centre for the Unknown, Lisbon, Portugal.
}

\begin{abstract}
Aberrant activation of telomerase in human cancer is achieved by various alterations within the TERT promoter, including cancer-specific DNA hypermethylation of the TERT hypermethylated oncological region (THOR). However, the impact of allele-specific DNA methylation within the TERT promoter on gene transcription remains incompletely understood. Using allele-specific next-generation sequencing, we screened a large cohort of normal and tumor tissues $(n=652)$ from 10 cancer types and identified that differential allelic methylation (DAM) of THOR is restricted to cancerous tissue and commonly observed in major cancer types. THOR-DAM was more common in adult cancers, which develop through multiple stages over time, than in childhood brain tumors. Furthermore, THOR-DAM was especially enriched in tumors harboring the activating TERT promoter mutations (TPMs). Functional studies revealed that allele-specific gene expression of TERT requires hypomethylation of the core promoter, both in TPM and TERT WT cancers. However, the expressing allele with hypomethylated core TERT promoter universally exhibits hypermethylation of THOR, while the nonexpressing alleles are either hypermethylated or hypomethylated throughout the promoter. Together, our findings suggest a dual role for allelespecific DNA methylation within the TERT promoter in the regulation of TERT expression in cancer.
\end{abstract}

\section{Introduction}

Telomeres are end chromosomal nucleoprotein structures that play critical roles in genome stability and cancer prevention (1). Due to the end-replication problem posed by the linear nature of human chromosomes, normal somatic cells, which lack means of telomere maintenance exhibit shortening of telomeres after each replicative cell cycle, ultimately leading to cellular senescence $(2,3)$. In contrast, cancer cells bypass cellular senescence and achieve replicative immortality by activating telomere maintenance (4). Telomerase activation via aberrant expression of the human telomerase reverse transcriptase (TERT) is the most prevalent telomere maintenance mechanism in human cancers (5). Multiple factors including transcriptional activators (6), TERT copy number variation (7), TERT promoter mutations (TPMs; refs. 8, 9), and hypermethylation at the TERT hypermethylated oncological region (THOR) in the

Conflict of interest: The authors have declared that no conflict of interest exists. Copyright: @ 2021, American Society for Clinical Investigation.

Submitted: December 15, 2020; Accepted: September 16, 2021; Published: November 1, 2021

Reference information: J Clin Invest. 2021;131(21):e146915

https://doi.org/10.1172/JCl146915. distal region of the promoter (10) have been identified as specific drivers of TERT expression in cancer. However, how these mechanisms independently or cooperatively activate TERT in the context of human tumorigenesis remains incompletely understood.

Allele-specific expression of TERT was previously shown to be common, mostly in the established cancer cell lines (11-15). At the transcriptional level, regulation of human gene expression can be modulated by trans- and/or cis-regulatory elements. While transregulatory elements (TRE) such as transcription factors usually affect both alleles, cis-regulatory elements (CREs) act on a single allele (16). In the context of the TERT promoter, TERT promoter mutations are an example of CRE, as these activating noncoding mutations (denoted as C228T and C250T occurring at chr5:1,295,228 and 1,295,250, GRCh37/hg19) often occur in a heterozygous manner. Several studies have demonstrated that heterozygous TPMs result in allele-specific expression of TERT in human cancer cell lines $(11,12,14)$. Interestingly, approximately $50 \%$ of the cancer cell lines exhibiting differential allelic expression (DAE) of TERT did not harbor TPMs (12). These observations strongly imply the existence of additional allele-specific alterations, other than TPMs, which may promote DAE of TERT.

DNA methylation of $\mathrm{CpG}$ dinucleotides within a gene promoter is an epigenetic modification often associated with gene silencing of 
Table 1. General characteristics of the human tissue samples

\begin{tabular}{|c|c|c|c|c|}
\hline Group & Type & $\begin{array}{c}\text { Samples with } \\
\text { HET pSNP }\end{array}$ & TPMs (\%) & $\operatorname{DAM}(\%)$ \\
\hline \multirow[t]{4}{*}{ Normal control } & Blood, ${ }^{A} n=47$ & 21 & $0 / 21(0)$ & $1 / 21(5)$ \\
\hline & Breast $^{,} n=20$ & 7 & $0 / 7(0)$ & $0 / 7(0)$ \\
\hline & Lungs, ${ }^{C} n=5$ & 3 & $0 / 3(0)$ & $0 / 3(0)$ \\
\hline & Prostate, ${ }^{A} n=5$ & 3 & $0 / 3(0)$ & $0 / 3(0)$ \\
\hline \multirow[t]{11}{*}{ Tumors } & Bladder, ${ }^{0} n=24$ & 10 & $9 / 10(90)$ & $6 / 10(60)$ \\
\hline & Brain & & & \\
\hline & Ependymoma, ${ }^{A} n=68$ & 28 & $0 / 28(0)$ & $4 / 28(14)$ \\
\hline & Glioma, ${ }^{\mathrm{E}} n=48$ & 21 & $16 / 21(76)$ & $14 / 21(67)$ \\
\hline & Medulloblastoma, ${ }^{A} n=63$ & 25 & $1 / 25(4)$ & $6 / 25(24)$ \\
\hline & Meningioma, ${ }^{\mathrm{F}} n=133$ & 78 & $13 / 78$ (17) & $14 / 78$ (18) \\
\hline & Breast $^{\mathrm{B}} n=59$ & 35 & $0 / 35(0)$ & $12 / 35$ (34) \\
\hline & Colon, ${ }^{6} n=100$ & 47 & $0 / 47(0)$ & $15 / 47(32)$ \\
\hline & Lungs, ${ }^{C} n=23$ & 14 & $1 / 14(7)$ & $3 / 14(21)$ \\
\hline & Prostate, ${ }^{\mathrm{H}} n=23$ & 13 & $0 / 13(0)$ & $1 / 13(8)$ \\
\hline & Skin, ${ }^{A} n=34$ & 15 & $11 / 15(73)$ & $8 / 15$ (53) \\
\hline Total, tumors & $n=575$ & 286 & & $83 / 286$ (29) \\
\hline
\end{tabular}

${ }^{A}$ The Hospital for Sick Children. ${ }^{B}$ Princess Margaret Cancer Centre, University Health Network. 'Ontario Institute for Cancer Research (OICR). DUniversity Hospital Zurich. EDuke University Medical Center. ${ }^{\mathrm{F} T o r o n t o}$ Western Hospital, University Health Network. ${ }^{\circ}$ Ontario site of the Colon Cancer Family Registry (OFCCR). ${ }^{H}$ Medical University of Innsbruck.

the allele it is present on $(17,18)$. Previous studies from our group and others have identified and characterized the existence of cancerspecific hypermethylation at the THOR (Chr5:1,295,321 - 1,295,753, GRCh37/hg19) - distal to the core TERT promoter - in multiple human cancer types (10). In cancer cell lines, THOR was shown to have a repressive function on TERT expression in its unmethylated state, while THOR hypermethylation was shown to be sufficient to counter this repressive effect (10). As such, THOR hypermethylation may act as a cis-acting epigenetic modifier, accounting for DAE of TERT expression in human cancers.

In this study, we uncover the phenomenon of differential allelic methylation of THOR (THOR-DAM) and characterize its extent in multiple human cancers. We show that THOR-DAM is not a random process and is enriched in cancers with TPM in an allelespecific manner. Lastly, we functionally characterize the interaction between hypermethylation of THOR, the core promoter of TERT and the presence of TPM, suggesting a new model for allele-specific activation of TERT transcription in cancer.

\section{Results}

THOR-DAM in human cancer. To investigate the existence of allele-specific DNA methylation of the TERT promoter, we assessed 575 tumor samples from various tissues and 77 normal tissue samples (Table 1). We targeted a single region within THOR (Chr5:1,295,321 - 1,295,393, covering 7 CpG sites within THOR) (red bar, Figure 1A) surrounding a common single nucleotide polymorphism (rs2853669, referred to as promoter SNP or pSNP, Chr5:1,295,349, GRCh37/hg19). This region is within the proximal THOR (overlapping with the core TERT promoter), which was previously shown to have a regulatory function on TERT expression (10). Non-allele-specific THOR methylation data for the entire cohort are available in Supplemental Figure 1 (supplemental material available online with this article; https://doi.org/10.1172/JCI146915DS1).

From the initial cohort of samples, 286 tumors and 34 normal tissues harbored the heterozygous pSNP (alleles harboring "A" SNP or "G" SNP). Next-generation sequencing (NGS) reads (see Methods for sequencing platform details) from each sample were segregated based on the pSNP status to investigate allele-specific DNA methylation. We first analyzed the methylation difference between the " $\mathrm{A}$ " and "G" alleles (delta methylation) in all tumor types. In normal tissues, the methylation difference between alleles was not significant with a mean difference of $2.7 \%$. In contrast, the mean allelic methylation difference was more evident in human cancer, ranging between $3.0 \%$ in meningioma to $29.7 \%$ in colon cancer samples (Figure 1, B and C). There was no difference in the frequency of hypermethylation between the "A" and "G" alleles. The mean differential allelic methylation was significantly higher in tumor types such as colon and breast tumors $\left({ }^{* * *} P<0.0001\right.$ and ${ }^{*} P<0.05$, respectively), melanoma, bladder, and gliomas $\left({ }^{* * *} P<0.0001\right)$ compared with normal tissue.

To define and assess prevalence of THOR-DAM, we stringently categorized a sample to exhibit THOR-DAM if a tissue met both of the following criteria: (a) one allele is hypermethylated (>16.0\%) and the other allele is hypomethylated $(<16.0 \%)$ as previously established to be associated with TERT expression in cancer (ref. 10 and Figure 1C), and (b) the tumor sample has a mean methylation difference of $>8.2 \%$ (see Methods) between the hypo- and hypermethylated alleles. Using these criteria, tumors were categorized into either THOR-DAM or non-DAM (red and blue pies, respectively, Figure 1D). THOR-DAM was rarely observed in the normal tissue control $(3 \%, 1 / 34)$. Interestingly, we found that THOR-DAM is commonly observed in human cancer, with the overall prevalence of THOR-DAM being significantly higher at $29.0 \%(83 / 286)(P=0.02)$ (Table 1$)$.

THOR-DAM was nonrandomly distributed between cancer types. In cancers with short time of onset, including childhood cancer (i.e., medulloblastoma and ependymoma) and meningioma, the prevalence of THOR-DAM (14\%-25\%) and hypermethylation (8\%-20\%) was on the lower end of the spectrum (Figure 1D). In contrast, cancers known to develop from low grade (premalignant) to high grade (malignant), including lung, colon, and breast cancer, had higher prevalence of THOR-DAM (21\%, 32\%, and 36\%, respectively) and hypermethylation ( $>50 \%$ in all types) when compared with the tumor types with short time of onset. Interestingly, prostate cancer showed remarkably low prevalence of THOR-DAM (8\%) exhibiting biallelic THOR hypermethylation (Figure 1C) and high prevalence of THOR hypermethylation (69\%). These observations further support the hypothesis that THOR methylation is probably a dynamic, step-wise process $(19,20)$. Short-onset tumors exhibit lower level of overall THOR methylation, with longer-onset tumors starting to develop THOR hypermethylation on one allele, leading to higher prevalence of DAM and eventually leading to biallelic THOR hypermethylation, as observed in prostate cancer (19). 
A
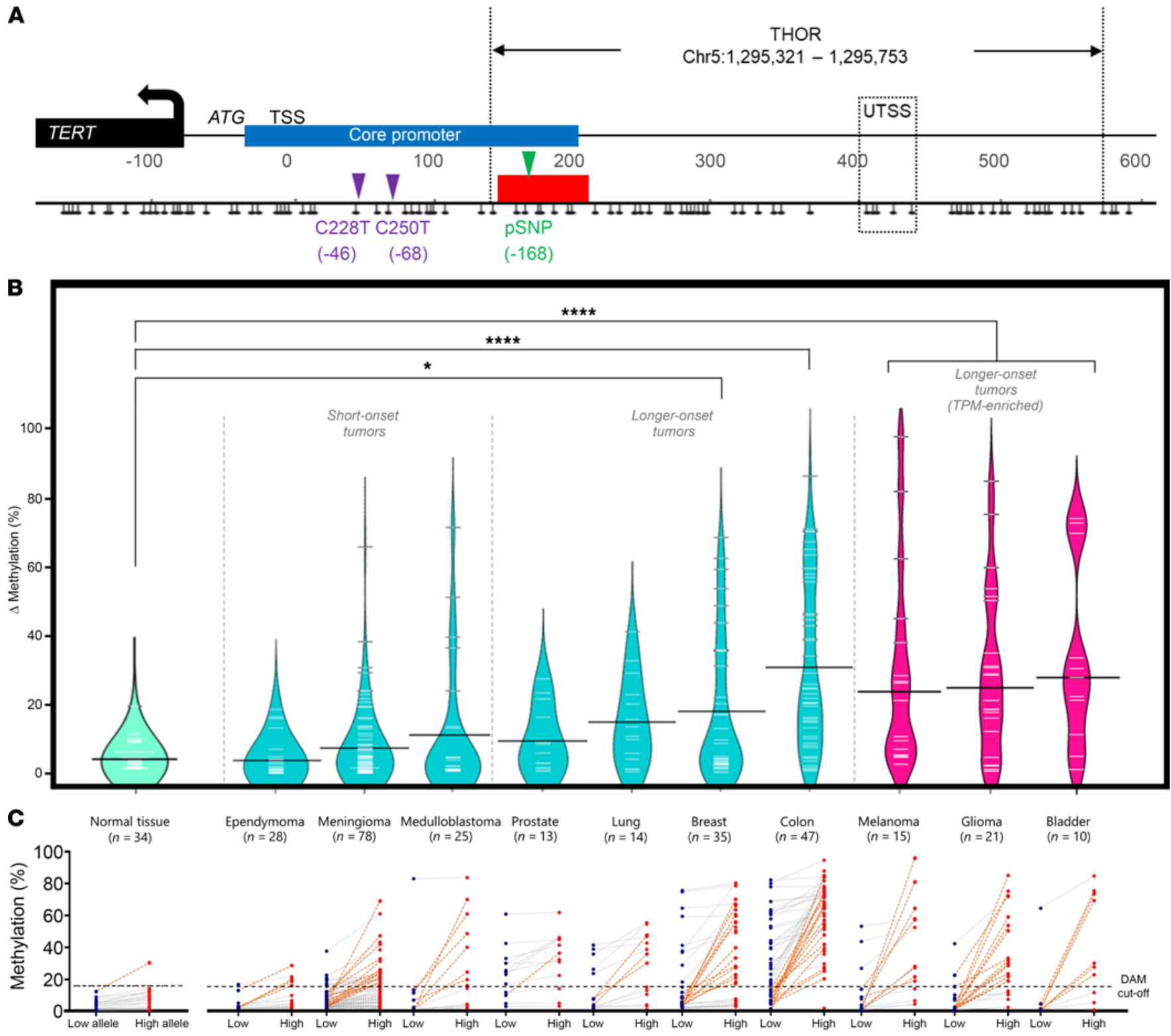

D

Normal tissue

Ependymoma Meningioma Medulloblastoma Prostate

Lung

Breast

Colon
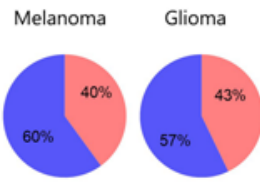

Bladder

THOR

status
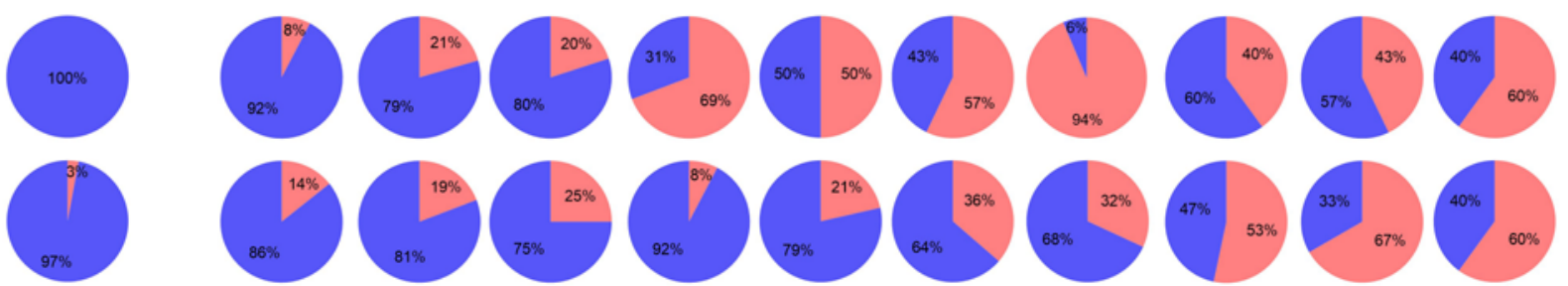

DAM
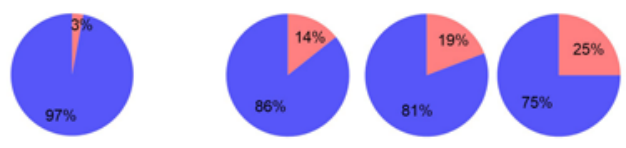

Figure 1. Prevalence of THOR-DAM in human cancer. (A) Schematic of the TERT promoter. Black lollipops represent $\mathrm{CpG}$ sites. Red bar indicates the region of DAM assessment within THOR. (B) Bean plot displaying the distribution of the allelic methylation difference in each tumor type. Each white line shows a methylation difference between the low- and high-methylated alleles in a sample, with the mean difference for each tumor type shown in a black line. Turquoise bars and pink bars indicate the tumor types with low and high prevalence of TPMs based on the previous literature (31). (C) Difference in methylation levels of the alleles separated with pSNP (A vs. G SNPs, rs2853669) within each tumor sample. Each pair of blue and red dots represents a tumor sample. Blue dots and red dots represent methylation levels of lower and higher methylated alleles, respectively. Orange dotted lines indicate the samples that are THORDAM. (D) Pie graphs showing prevalence of DAM and THOR hypermethylation in each tumor types (red = presence of attribute, blue = absence of attribute). 
Tumors where TPM is more prevalent include melanoma, adult glioma, and bladder cancer. These tumors exhibit the greatest mean allelic methylation difference (pink bean plots, Figure 1B) and the highest prevalence of THOR-DAM (53\%, 67\%, and 60\% respectively) (Figure 1, C and D) suggesting that THOR-DAM may be enriched in the context of TPM.

THOR-DAM in the context of TPM. To further explore THORDAM in the context of TPM, we first segregated all tumor samples based on their TPM status (WT vs. TPM) and assessed the prevalence of THOR-DAM in the 2 groups. The prevalence of THOR-DAM in the tumors harboring TPMs was significantly higher (51\%, 26/51) compared with the WT tumors $(24 \%, 56 / 235)$ (Figure $2 \mathrm{~A}$ ) ( $\chi^{2}$ test, $P$ $\left.=9.87 \times 10^{-5}\right)$. We then hypothesized that this observation would be consistent within each tumor type. Glioma $(n=21)$ and meningioma $(n=78)$ were the 2 tumor types with a sufficient number of samples for statistical comparison between WT and TPM groups (Figure 2B). All samples in the glioma group were high-grade glioblastoma (GBM) samples. In these tumors, the presence of TPMs trends with higher prevalence of THOR-DAM ( $\chi^{2}$ test, $P=0.15$ and $P=0.19$ in glioma and meningioma subgroups, respectively). The other tumor types were either dominantly WT or TPM (Supplemental Figure 2), with the latter group (i.e., melanoma and bladder cancer) exhibiting greater than $50 \%$ prevalence of THOR-DAM, in contrast to the dominantly WT tumor types where none of them exceeded 35\%.

Taking advantage of the NGS technology where an individual sequencing read reflects the DNA methylation pattern of an individual DNA molecule pertaining to a single TERT allele, we looked further into allele-specific methylation of THOR with analysis of individual sequencing reads in our glioma subcohort $(n=21)$. Red and yellow colors were used to describe relative methylation levels between the 2 alleles, where red color describes an allele with higher methylation and yellow color describes an allele with lower methylation. WT gliomas exhibited a similar pattern of allelic methylation where both alleles were lowly methylated (Figure 2C). In the majority (80\%, 4/5) of WT gliomas, the frequency of sequencing reads with most ( $\geq 5$ of 7 ) of the $\mathrm{CpG}$ sites methylated in the same read was less than $1 \%$ in both alleles (Figure 2C and Supplemental Figure 3), suggesting biallelic hypomethylation of proximal THOR (which is part of the core TERT promoter based on our previous functional analysis; ref. 10).

In contrast, in 50\% (8/16) of TPM-harboring gliomas, the frequency of sequencing reads with most ( $\geq 5$ of 7 ) CpG sites methylated ranged between $20 \%-87 \%$ in the non-TPM ("A") allele (Figure 2C and Supplemental Figure 3). Meanwhile, the TPM ("G") allele displayed high frequency ( $\geq 75 \%$ ) of completely unmethylated sequencing reads ( 0 CpG sites methylated in the same read) (Figure $2 \mathrm{C}$ and Supplemental Figure 3). Taken together, our data reveal that there is a nonstochastic hypermethylation of the TERT promoter, and THOR-DAM is more prevalent in tumors where a TPM is present.

THOR-DAM and allele-specific TERT expression. To investigate the functional impact of THOR-DAM on allele-specific expression of TERT in the context of both TPM and non-TPM, we first selected 11 cancer cell lines (5 TPM and $6 \mathrm{WT}$ ) with a combination of heterozygous SNPs in both the promoter (pSNP, rs2853669) and the exon 2 (exSNP, rs2736098; Table 2). Human cancer cell lines were chosen as a model for investigating the association between the allele-specific methylation (based on pSNP) and the allelespecific TERT expression (based on exSNP), as cancer cell lines mimic
THOR methylation characteristics of human cancers (10). We used the amplification refractory mutation system (ARMS) PCR (21) to determine the allele-specific association of pSNP (A or G, used for distinction of allele-specific methylation) and exSNP (C or T, used for distinction of allele-specific expression). With exception of LnCAP, all cell lines exhibited monoallelic expression of TERT. Using our targeted NGS sequencing tool (10), we assessed the allele-specific methylation pattern of both the core TERT promoter (blue bar on schematic, Figure 3) (Chr5:1,295,313 - 1,295,395, GRCh37/hg19) and the proximal region of repressive THOR (rTHOR, Chr5:1,295,395 $-1,295,524$, GRCh37/hg19) - a genomic region within THOR that previously showed repressive effect on a gene promoter (10).

In TPM cancer cells, the allele harboring heterozygous TPM (yellow line) was hypomethylated within the core TERT promoter and hypermethylated in the distal rTHOR with mean methylation level of $35 \%$, exceeding the hypermethylation cut-off of $16 \%$ (Figure 3A). Importantly, the non-TPM allele (red line) was hypermethylated throughout the core TERT promoter region and the rTHOR and did not display any TERT transcriptional activation. This was consistent in all TPM cancer cell lines screened $(n=5$, Supplemental Figure 4$)$.

Cancer cell lines with a WT TERT promoter and differential allelic expression of TERT exhibited a similar pattern of THOR methylation. The core TERT promoter displayed a lower level of methylation in the allele that exhibited monoallelic expression of TERT (yellow line, Figure 3B). The mean fold-difference of the core TERT promoter methylation between the low- and high-methylated alleles was 1.7-fold. In contrast, in the distal rTHOR, hypermethylation was observed in both alleles (Figure 3B). The difference in methylation between the expressing and nonexpressing alleles in each of the WT cancer cell lines $(n=5)$ are shown in Supplemental Figure 5.

Meanwhile, WT cancer cell lines with biallelic TERT expression revealed hypomethylation of both alleles within the core TERT promoter and hypermethylated rTHOR (Figure 3C). These data suggest that the DNA methylation status of different areas within the TERT promoter can result in differential allelic expression of TERT. DNA methylation within the TERT promoter appears to have a dual role (i.e., either activation or repression) depending on the region being methylated (i.e., core TERT promoter vs. rTHOR).

Functional impact of THOR hypermethylation based on TPM and core promoter status. To functionally characterize the dual role of DNA methylation within the TERT promoter on allele-specific TERT expression, we performed reporter gene (CpG-free backbone) assays in TPM (LN229) and non-TPM (HT1080) cancer cell lines. The experiment was performed in both TPM and non-TPM contexts to control for potential variables associated with the different cellular contexts, including the presence of different transcription factors, specifically in the TPM cell lines, that may act upon the de novo TPMs.

The addition of unmethylated THOR upstream to the core TERT promoter resulted in approximately 2-fold reduction of the reporter gene expression, consistent with previous findings suggesting that unmethylated THOR may act as a repressive element of TERT expression (Figure 4, A and C, empty red vs. blue bars; ref. 10). To examine the effect of THOR methylation with an unmethylated proximal core promoter, we used our previously tested CpGfree $h E F 1$ gene promoter (Figure 4, B and D, red vs. blue checkered bars on bottom graphs for each cell line) (10). This experiment was performed in place of targeted in vitro methylation of THOR in the 
A

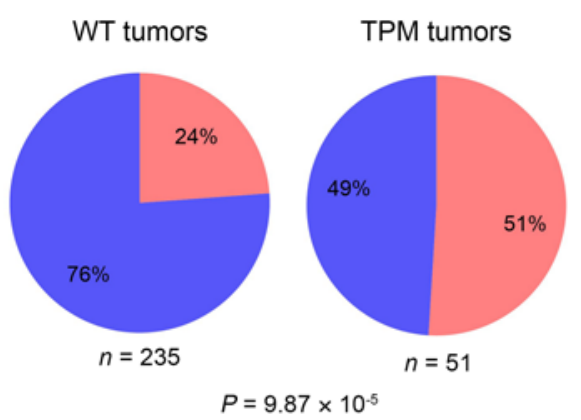

B

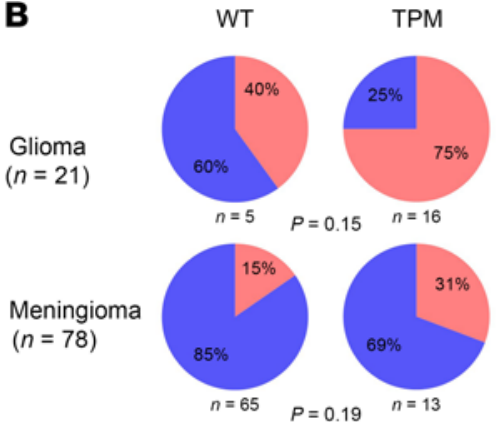

C
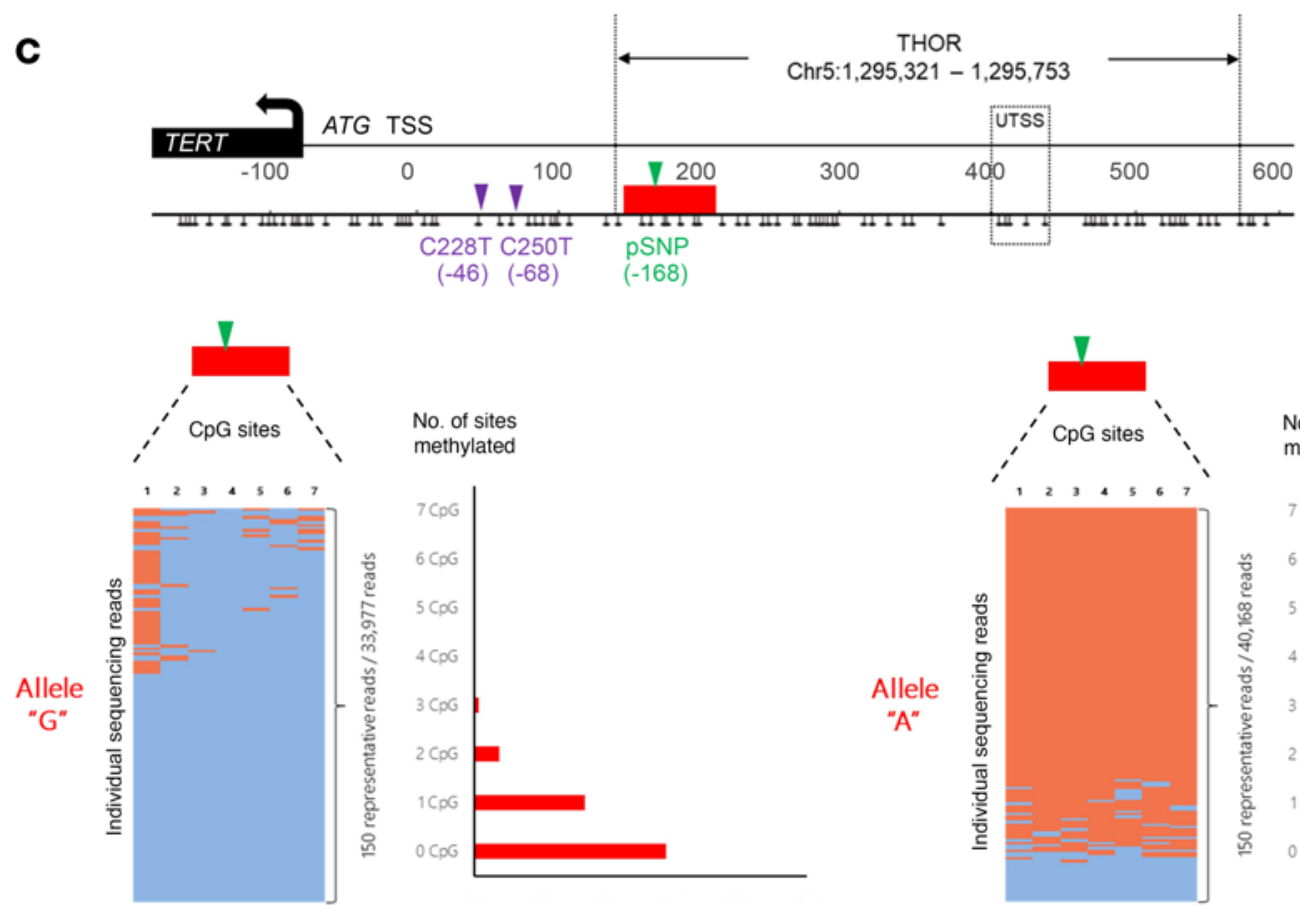

No. of sites
methylated

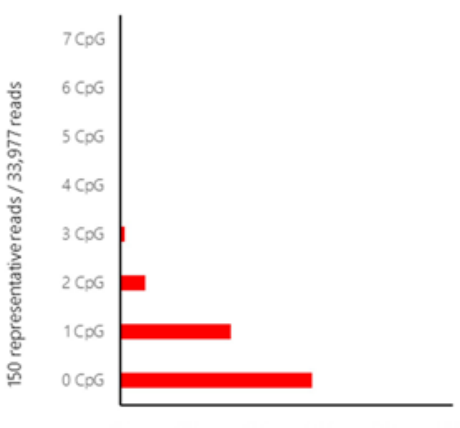

No. of sites
methylated

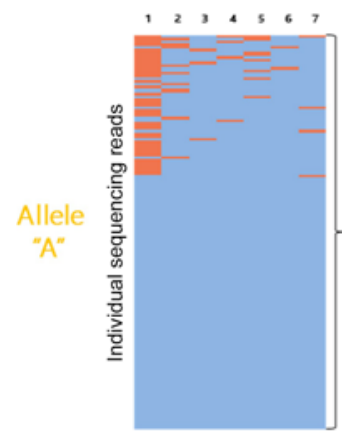

D555

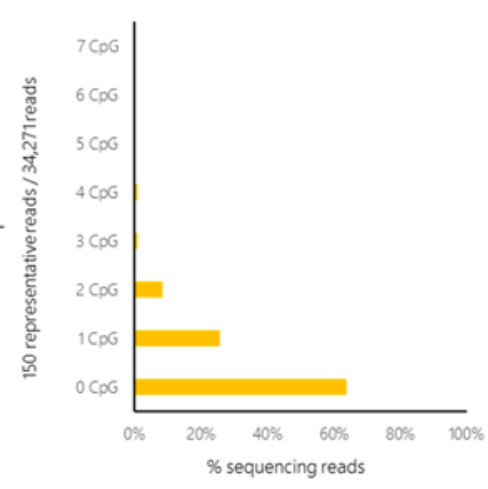

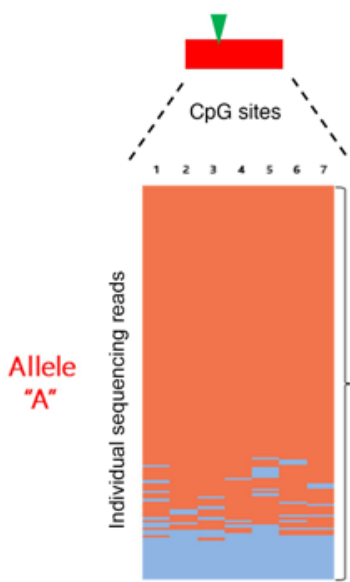

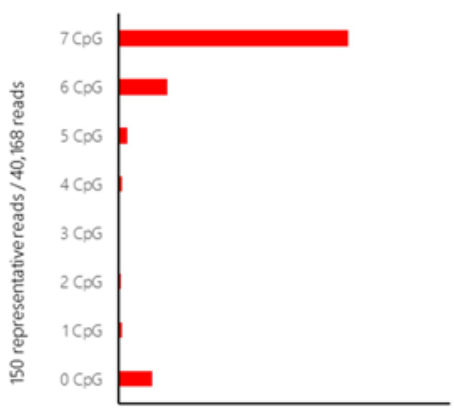

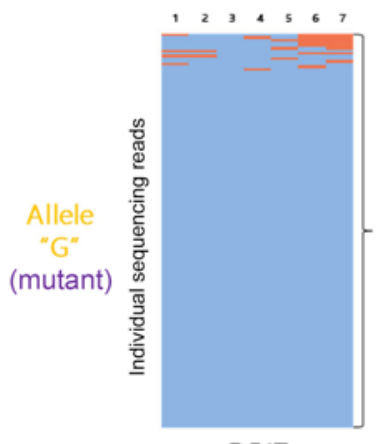

D517
(TPM tumor)

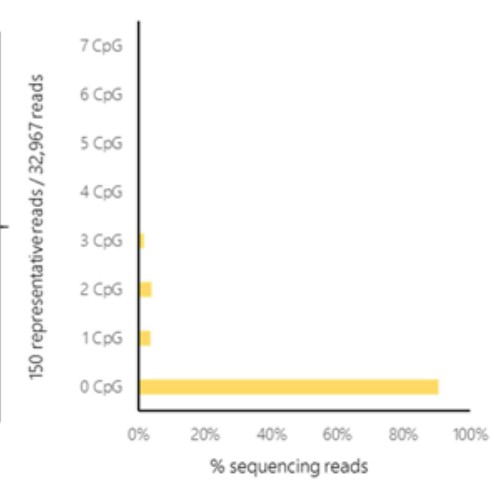

Figure 2. Association between THOR-DAM and TPM in human cancer. (A) Pie chart indicating the prevalence of THOR-DAM signature in the context of WT and TPM cancers. Blue and red pies indicate presence/absence of attribute respectively. (B) Pie chart indicating the prevalence of THOR-DAM signature in the context of WT and TPM cancers in glioma $(n=21)$ and meningioma $(n=78)$. Blue and red pies indicate presence/absence of an attribute, respectively. (C) Individual sequencing read analysis of allele-specific THOR methylation status in a representative WT cancer (D555) and TPM cancer (D517). Heatmap shows a representative subset of sequencing reads (150 reads) that reflect the overall trend (raw read numbers: D555, 33,977 and 34,271 reads in alleles with pSNP A and G, respectively; D517, 32,967 and 40,168 reads in alleles with pSNP G and A, respectively). Each line of the heatmap represents a sequencing read. Blue and orange indicate unmethylated and methylated $\mathrm{CpG}$ sites (1-7) across the region of analysis. Bar graphs indicate the proportion of sequencing reads separated based on the number of methylated $\mathrm{CpG}$ sites within each read. 
Table 2. General characteristics of the human cancer cell lines

$\begin{array}{lccccc}\text { Cell line } & \text { Type } & \text { TPM } & \text { pSNP } & \text { exSNP } & \begin{array}{c}\text { Allele-specific TERT } \\ \text { expression }\end{array} \\ \text { LN-229 } & \text { Clioblastoma } & \text { C228T } & \text { Y } & \text { Y } & \text { Monoallelic } \\ \text { U-118 } & \text { Clioblastoma } & \text { C228T } & \text { Y } & \text { Y } & \text { Monoallelic } \\ \text { U-87 } & \text { Clioblastoma } & \text { C228T } & \text { Y } & \text { Y } & \text { Monoallelic } \\ \text { ONS76 } & \text { Medulloblastoma } & \text { C228T } & \text { Y } & \text { Y } & \text { Monoallelic } \\ \text { UW-228 } & \text { Medulloblastoma } & \text { C228T } & \text { Y } & \text { Y } & \text { Monoallelic } \\ \text { LnCAP } & \text { Prostate cancer } & \text { WT } & \text { Y } & \text { Y } & \text { Biallelic } \\ \text { HCT15 } & \text { Colorectal cancer } & \text { WT } & \text { Y } & \text { Y } & \text { Monoallelic } \\ \text { LS513 } & \text { Colorectal cancer } & \text { WT } & \text { Y } & \text { Y } & \text { Monoallelic } \\ \text { PANC10.05 } & \text { Pancreatic cancer } & \text { WT } & \text { Y } & \text { Y } & \text { Monoallelic } \\ \text { RPMI8226 } & \text { Myeloma } & \text { WT } & \text { Y } & \text { Y } & \text { Monoallelic } \\ \text { SNU-C1 } & \text { Colorectal cancer } & \text { WT } & \text { Y } & \text { Y } & \text { Monoallelic }\end{array}$

context of core TERT promoter, as in vitro methylation cannot be targeted to a specific region of an insert. Remethylation of THOR resulted in an increased expression of the construct with an unmethylated core promoter, implying that THOR hypermethylation counters the repressive effect of unmethylated THOR.

The introduction of TPMs to the core promoter was sufficient to promote reporter gene expression even in the presence of unmethylated THOR, implying the potency of TPMs as a strong cis-regulatory activator (Figure $4 \mathrm{C}$, empty red bar vs. empty purple bar). Nevertheless, hypermethylation of the core TERT promoter completely abolished reporter gene expression regardless of THOR methylation status or the presence of TPM. These findings were consistent in both cancer cell lines tested (Figure 4, A and C, empty vs. solid bars). Together, the functional and mechanistic experiments (Figure 3 and Figure 4) further suggest a dual role of allele-specific hypermethylation within the TERT promoter. THOR hypermethylation enhances TERT expression when coupled with a hypomethylated proximal core promoter, while hypermethylation of the core TERT promoter abolishes the impact of activating alterations including both THOR hypermethylation and TPMs (Figure 5).

\section{Discussion}

The presence of hypermethylation in the distal part of the TERT promoter - more specifically at THOR - in TERT-expressing cancers has been observed in multiple cancer types (10, 19, 20, 22-25). Although THOR hypermethylation in cancer is associated with increased TERT expression (10), the impact of TERT promoter allele-specific hypermethylation on TERT expression is still relatively unknown. This study reveals the first evidence of allele-specific hypermethylation of THOR in multiple tumor types and provides insight into the functional impact of DNA methylation in different regions within the TERT promoter on TERT expression in cancer.

THOR-DAM has not been previously described in cancer tissues. Our data suggest that THOR-DAM is prevalent in human cancer. Importantly, the degree of THOR-DAM varies between tumor types. Tumors that are expected to have fewer cell divisions or less time from tumor initiation to cancer presentation (e.g., childhood ependymoma and medulloblastoma; ref. 26) exhibit low prevalence of TPMs and low THOR-DAM. Adult carcinomas that do not commonly have TPMs but have high THOR methylation (i.e., lung, breast, and colon cancers) exhibited higher prevalence of DAM. In these tumors, the absence of TPMs as a potent cis-regulatory element to drive TERT expression indicates that a different mechanism - involving hypermethylation of THOR and modulation of its repressive effects - may be selected for during the process of tumorigenesis. The higher prevalence of DAM is likely due to higher variability of DNA methylation pattern between the 2 alleles and possibly between different cells when overall THOR methylation is higher, and may also suggest a dynamic, step-wise process of DNA methylation. We speculate that higher prevalence of biallelic THOR hypermethylation in prostate cancers, which develop over years of premalignant transformation and are present in at least one-third of men over 50 years of age (27), is an indication of stabilized telomerase activation through THOR hypermethylation, while "younger tumors" have more heterogeneous DAM.

An interesting finding consistent in both human cancer samples and cell lines was the enrichment of THOR-DAM in the context of TPMs. The prevalence of DAM is highest in tumor types that exhibit frequent TPMs, including glioma, bladder cancer, and melanoma. In addition, even within a cancer type, significant enrichment in THOR-DAM exists in TPM-harboring tumors (Figure 2B). These findings are in agreement with previous studies that report allele-specific DNA methylation of the TERT promoter in TPM cancer cell lines (13) and are further validated in our cell line data.

Since both THOR and TPM are allele-specific alterations of the TERT promoter, we speculated that it may have a functional effect on DAE of TERT. This phenomenon was reported prior to the discovery of TPMs (15), but the discovery of heterozygous TPMs only accounted for approximately $50 \%$ of TERT DAE observed in human cancer cell lines. Nearly $50 \%$ of cancer cell lines exhibiting TERT DAE were in fact WT cancer cell lines (12), implying the presence of additional cis-regulatory element(s) yet to be uncovered. In this study, we show that differential allelic methylation of the core TERT promoter (a part of proximal THOR) may be involved in DAE of TERT in the context of both WT and TPM cancer cells. In all cancer cell lines we tested, the expressing allele exhibited low methylation of the core TERT promoter and hypermethylated rTHOR. The impact of locus-specific methylation was validated in our functional study, where the hypermethylation of core TERT promoter resulted in a complete abolishment of reporter gene expression despite the presence of TPMs and/or THOR hypermethylation.

Based on the clinical, functional, and modeling data in this study, we propose the following role for epigenetic control of the TERT promoter in cancer (Figure 5). Hypomethylation of the core TERT promoter appears to be a prerequisite for all cells (and alleles) to express TERT. Indeed, hypermethylation of the core promoter will result in a lack of expression from the specific allele (Figure 3 and Figure 4). However, since normal cells that lack TERT expression also exhibit hypomethylation of the core promoter, this is probably necessary but not sufficient to activate TERT expression in cancer. THOR hypermethylation would provide a mechanism for cancer cells to upregulate TERT expression during malignant transformation. 


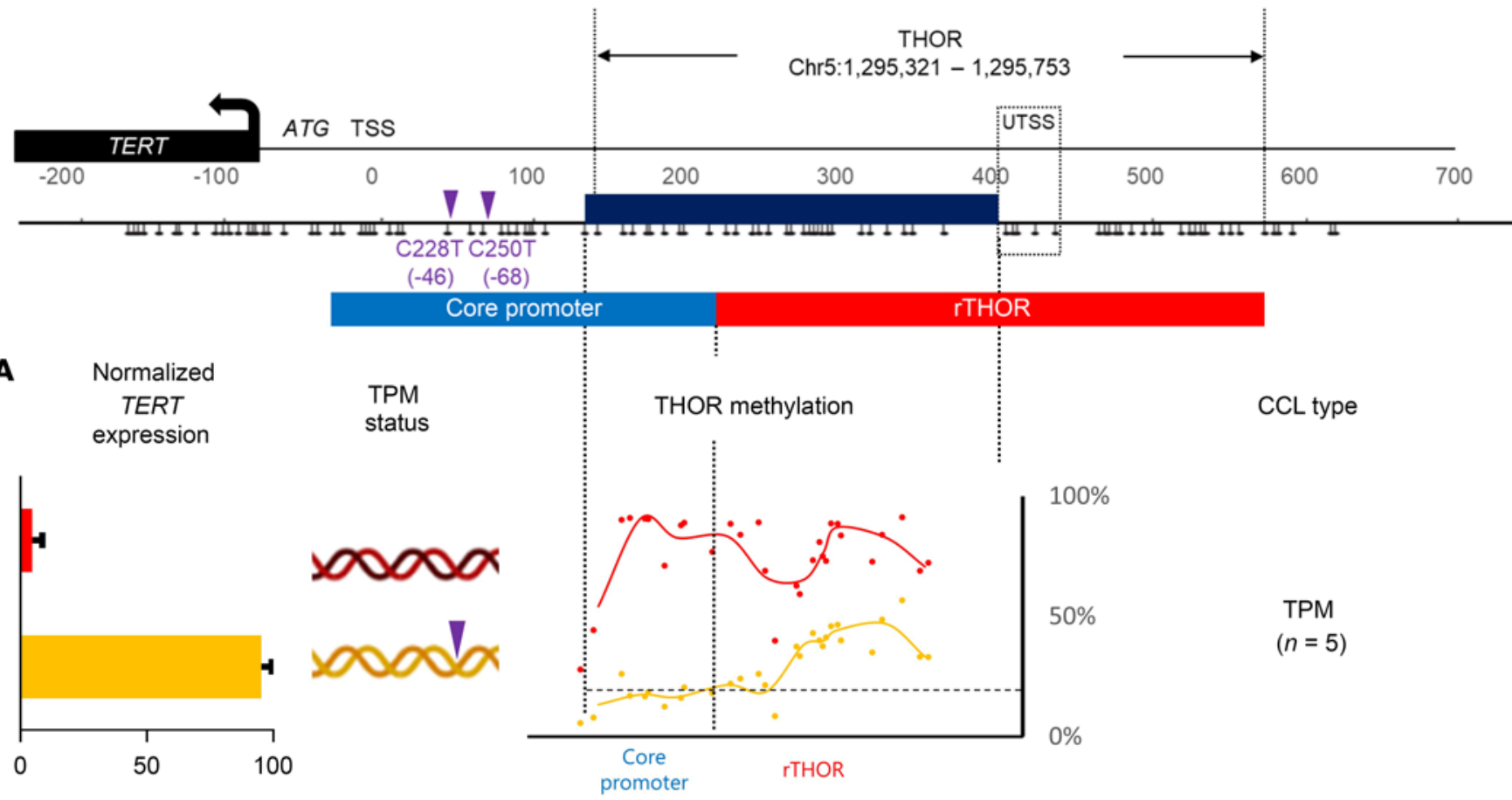

Fold-
difference
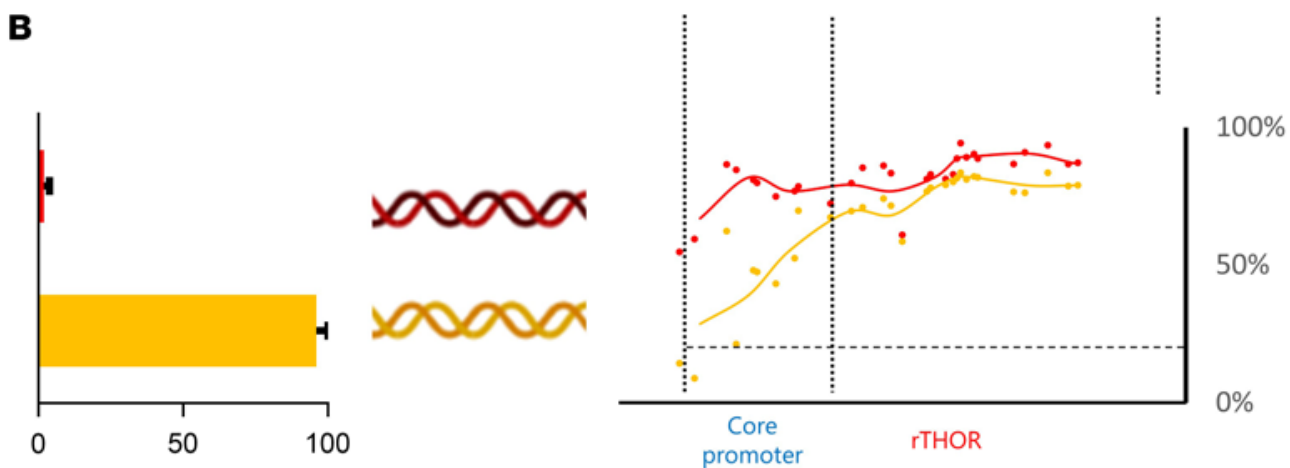

Fold-

1.7-fold $\quad 1.1$-fold
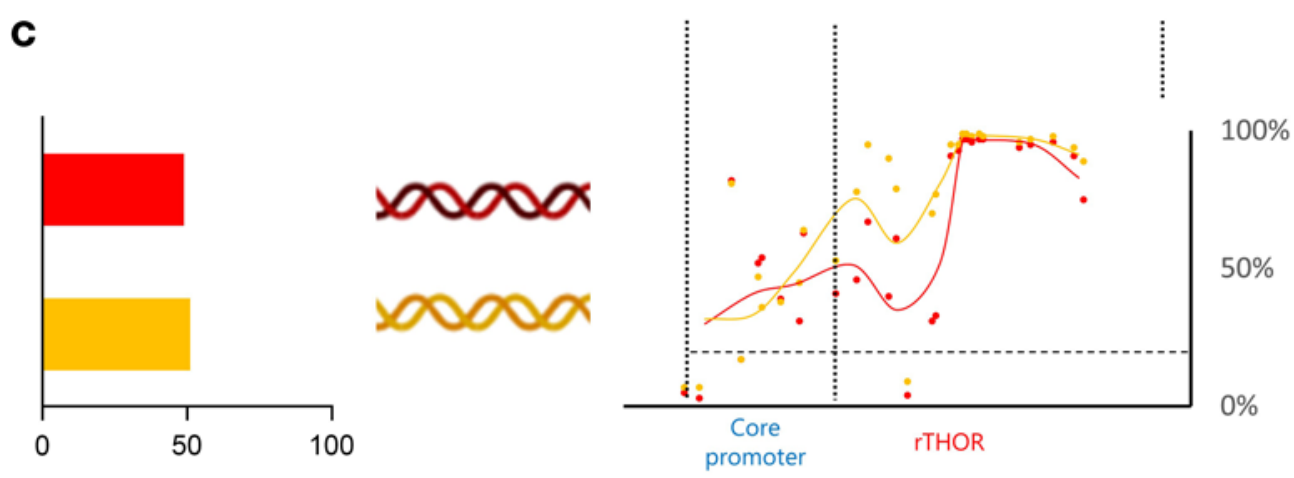

WT DAE

$(n=5)$

Folddifference

1.1-fold

1.1-fold

WT BAE

$(n=1)$

Figure 3. Allele-specific CpG methylation patterns of the TERT promoter in TPM and WT cancer cell lines. Red and yellow indicate each of the alleles separated based on the promoter SNP and exon SNP. Line graphs with dots (raw values) indicate average levels of allele-specific CpG methylation in the TERT promoter. Fold-differences are calculated based on the average of all $\mathrm{CpC}$ sites within each region. Position of purple triangle (TPMs) shows which allele harbors TPMs. Bar graphs show normalized allelic TERT expression. 
Our model provides explanation for TERT expression and telomere maintenance in normal and malignant cells. Normal cells lacking telomerase activity and nontelomerase-dependent cancer cells, such as tumors exhibiting alternative lengthening of telomeres (28), are hypomethylated throughout the TERT promoter. Noncancerous immortal stem cells including embryonic stem cells, hematopoietic stem cells, and induced pluripotent stem cells (iPSCs), all exhibit low levels of THOR methylation and a high level of TERT expression compared with normal cells (29), probably through OKSM (Oct4, Klf4, Sox2 and c-Myc) transcription factor-based induction of pluripotency and immortality.

During transformation, THOR becomes hypermethylated to promote reactivation of TERT and cellular immortality. Indeed, additional analysis of THOR methylation in a primary fibroblast cell line (WI38) and its corresponding, in vitro SV40 immortalized subline (VA13_2RA) has revealed THOR hypermethylation in the transformed cell line and hypomethylation in the nonimmortalized WI38 cell line, associated with a concomitant acquisition of TERT transcription (Supplemental Figure 6). Additional studies are needed to completely understand the relationship between THOR methylation and malignant transformation of cells.

In telomerase-dependent cancers, the most commonly observed cancer-associated TERT alterations are TPMs and THOR hypermethylation. In the absence of TPMs, most cancers will develop monoallelic or biallelic TERT expression by hypermethylation of THOR in conjunction with hypomethylation of the core TERT promoter. Hypermethylated core promoter will lead to complete abolishment of TERT expression based on our functional experiments. This mechanism is consistent in both WT and TPM-driven tumors. In agreement with these findings, analysis of 833 cancer cell lines from 23 different tissue types has also recently reported the upstream hypermethylation (THOR) and proximal core promoter hypomethylation as a commonly observed phenomenon (30). However, in TPM tumors, the mutant allele is always associated with hypomethylated core promoter and hypermethylated distal THOR, enhancing TERT expression in these cancers. Therefore, in a subset of tumors where TPMs are present, TERT promoter activation depends on these heterozygous mutations to drive TERT expression, as TPMs were shown to result in the highest levels of TERT promoter activation even in the absence of THOR hypermethylation (Figure 4). Our model provides an explanation for the discrepancies with the previous studies showing the correlation between low methylation of the TERT promoter and increased TERT expression $(13,30)$. Interestingly, THOR methylation is frequently lower in the TPM-harboring allele, although still at the hypermethylated level, possibly suggesting an ongoing process of hypermethylation as observed in several cancers $(19,20)$. Nevertheless, additional experiments are required to determine the mechanism of THORDAM in TERT activation during the process of tumorigenesis.

Overall, the data from recent studies focusing on differential allelic expression of TERT $(11-14,30)$ and the data in this study on THOR-DAM further expand our knowledge regarding the unique interaction between genetic and epigenetic alterations in the control of TERT activation in cancer. Additional studies analyzing the methylation levels at both the core promoter and THOR in larger patient tumor cohorts are needed to fully understand the causes and mechanism of unmethylated THOR as a repressive element, and whether it acts as a binding platform for other trans-regulatory factors involved in repression of TERT expression in cancer cells. However, it is plausible that while hypomethylation of the proximal TERT core promoter is essential for TERT expression, THOR hypermethylation may act as an enabler of sustained TERT expression, likely through regulating accessibility to cis-regulatory elements.

\section{Methods}

Patient tissues and cell lines. Tissue samples were collected from participating centers (Table 1). Cell lines used in the study were either obtained from collaborators or purchased through ATCC.

Genomic DNA/RNA preparation, bisulfite PCR, and NGS. Genomic DNA for tumor samples was obtained from collaborators. Cell line DNA and RNA were isolated using Qiagen AllPrep DNA/RNA Mini Kit (cata$\log 80204)$ and were stored at $-20^{\circ} \mathrm{C}$ until use. Genomic DNA (100 ng) was bisulfite converted using the EZ DNA Methylation kit from Zymo Research in accordance with the manufacturer's protocol. HotStarTaq Plus Master Mix kit from Qiagen was used to PCR amplify target amplicon from bisulfite-converted DNA using different primer sets. Importantly, the primers used to amplify the PCR amplicon submitted for NGS do not overlap CpG sites. This design aimed at avoiding introduction of PCR amplification biases by differential CpG methylation, leading to distortion of NGS results. The resulting PCR products for MiSeq (100 bp to $120 \mathrm{bp}$ amplicons) were prepared in accordance with the $16 \mathrm{~S}$ Metagenomic Sequencing Library Preparation guide (15044223 B, Illumina, Inc.) and were sequenced following the manufacturer's instructions for the MiSeq Reagent Kit v3 to obtain 125 nucleotide read lengths.

Sequencing read alignment and allele-specific methylation analysis. Sequence reads were identified using standard Illumina base-calling software. Adapter sequences were trimmed and sequencing reads containing at least one base with a Phred quality score below 20 were discarded prior to analysis. Resulting FastQ files were separated based on the promoter SNP (pSNP, rs2853669 G or A) status, using a customized python code. The separated FastQ files were aligned against the reference genome (GRCh37/hg19) using BS-Seeker2 (default parameters), then the resulting BAM/SAM files were mapped with Bowtie2. The methylation level of each sampled cytosine was calculated as the number of reads reporting a $\mathrm{C}$, divided by the total number of reads reporting either a $\mathrm{C}$ or $\mathrm{T}$.

We used a NGS strategy that shows DNA methylation pattern originating from each allele. Unlike traditional strategies that show average methylation values for each $\mathrm{CpG}$ site from sequencing reads of different alleles (down-the-read analysis), the NGS strategy allows for determination of a DNA methylation pattern originating from each allele (individual sequencing read analysis). Therefore, the generated data are more suitable for comparison of allelic DNA methylation pattern.

Defining the criteria for THOR-DAM. There were 2 criteria used to call a tumor sample to have THOR-DAM: (a) one allele is hypermethylated $(>16.0 \%)$ and the other allele is hypomethylated $(<16.0 \%)$ as previously established (10), and (b) the tumor sample has a mean methylation difference of greater than $8.2 \%$ between the low- and high-methylated alleles. This cut-off value for DAM was calculated by taking the mean delta methylation (between the low- and high-methylated alleles) of the normal blood samples $(n=21)$ and taking the value of 2 standard deviations above the mean. Calculated mean delta methylation $( \pm$ SD) of normal blood samples was $1.2 \%$ ( $\pm 3.5 \%)$. After adding 2 SDs to the average, the cut-off for THOR-DAM used in this study was $8.2 \%$. 


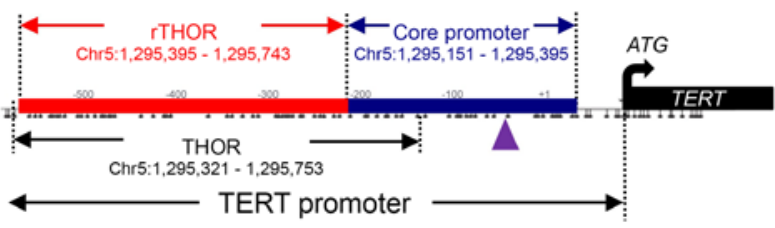

HT1080

A

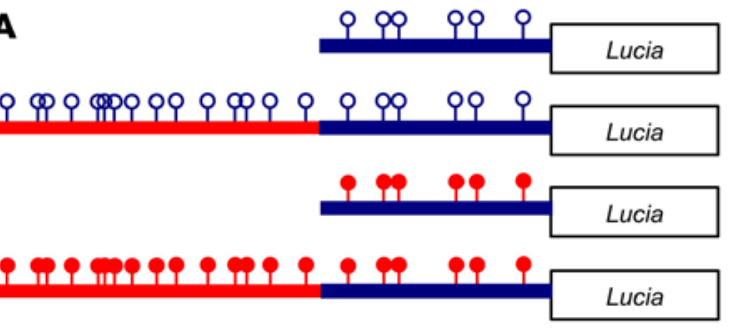

B
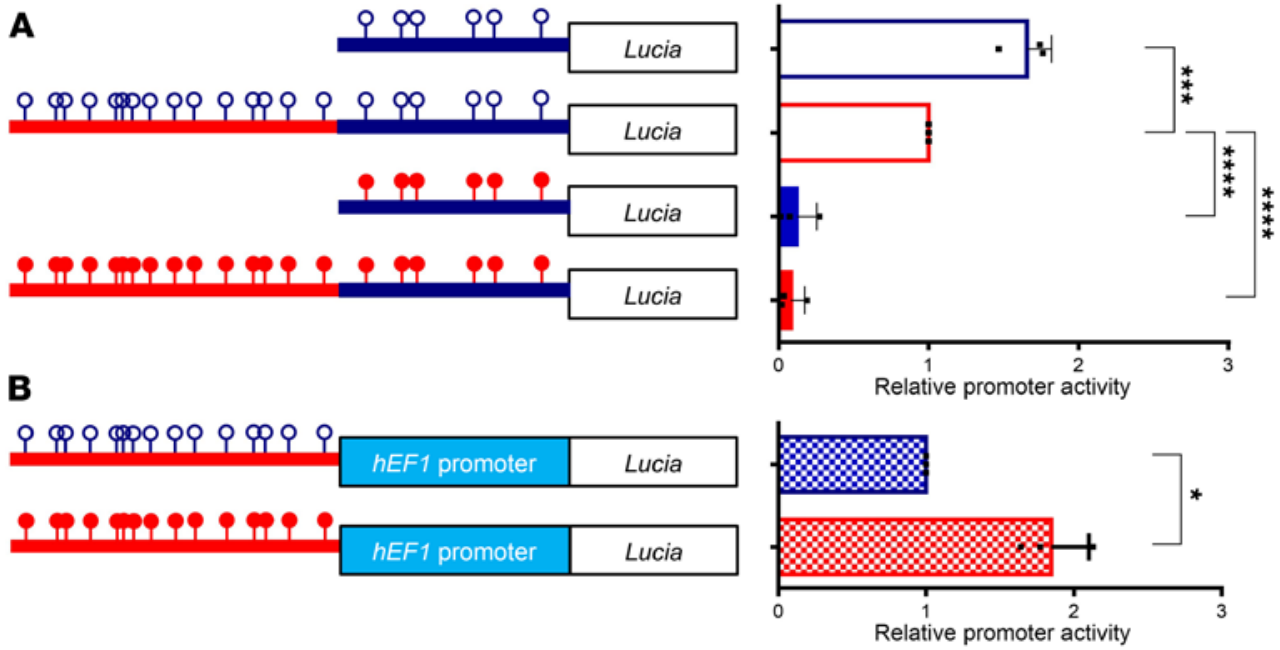

LN229

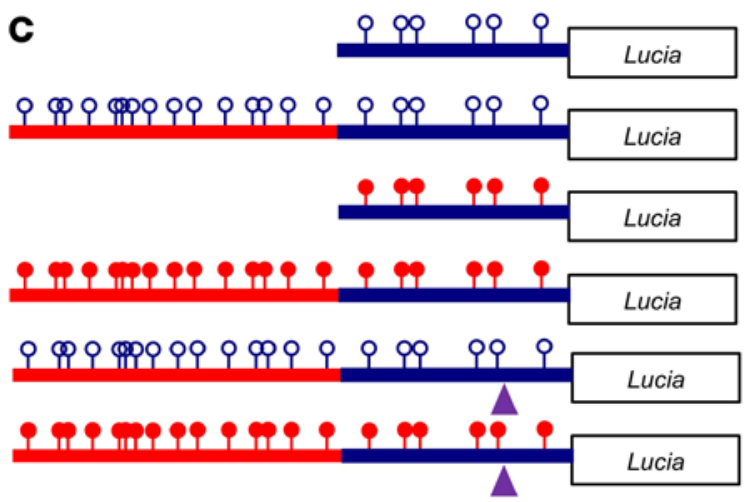

D
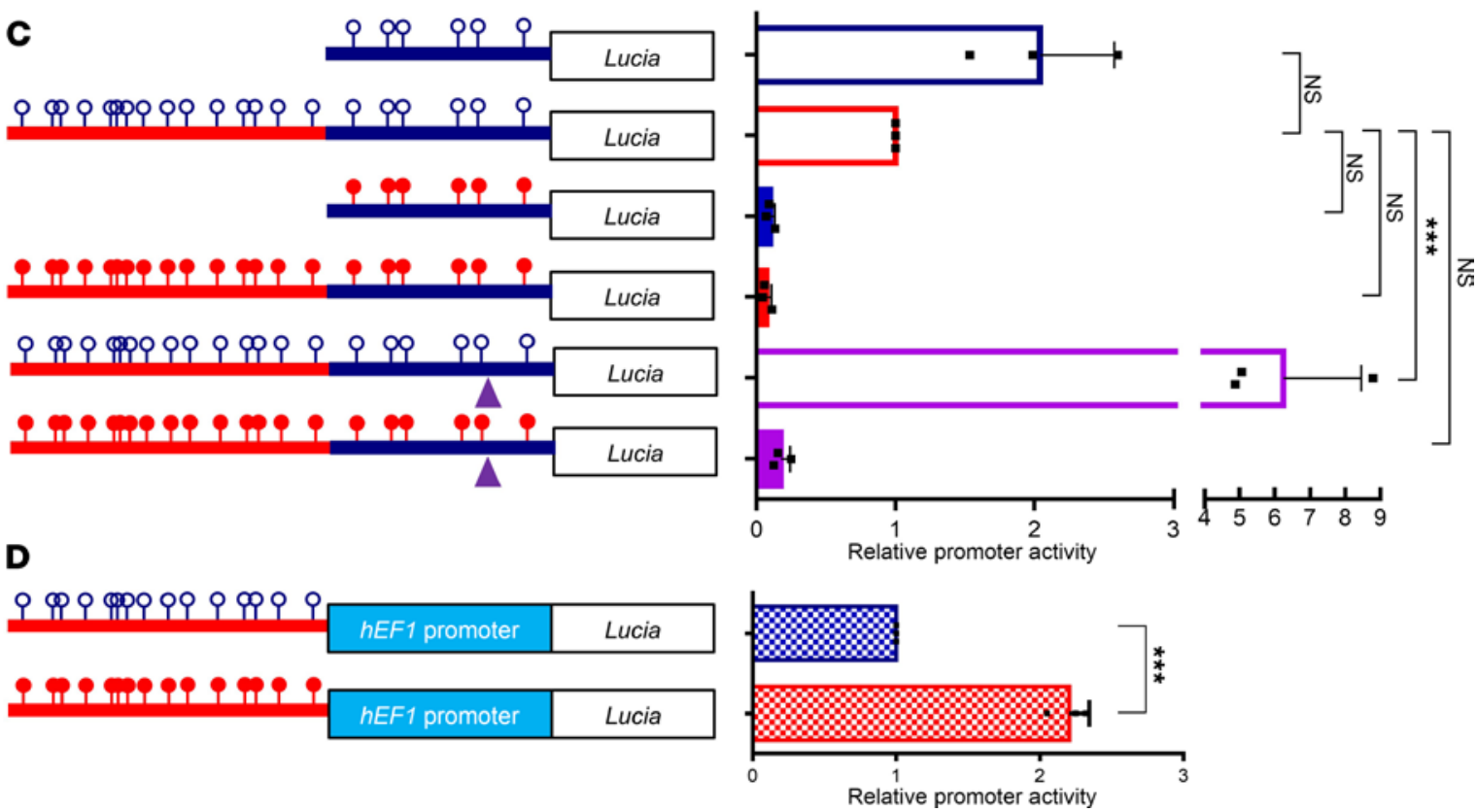

Figure 4. Impact of THOR and TPMs based on the core TERT promoter methylation status. (A and C) Effects of THOR addition to the core promoter region and effects of hypermethylation of both components of the TERT promoter on expression in HT1080 and LN229 cell lines respectively. (B and D) Effect of THOR-specific hypermethylation on TERT expression in both cell lines. For the data shown, each experiment was performed in triplicate. Purple triangle indicates the presence of C228T TPM. Blue and red lollipops indicate the unmethylated and methylated CpG Normalized fold changes in TERT or hEF1 promoter activity are shown for the specified CpG-free Lucia reporter constructs transfected into a non-TPM (HT1080, A and B) and TPM (LN229, C and D) cancer cell lines. One-way ANOVA with Dunnett's post hoc test for multiple comparisons was used for statistical analysis (***adjusted $P<0.001$, ${ }^{* * * *}$ adjusted $P<$ 0.0001). For checkered bar graphs (B and $\mathbf{D}$ ), normalized fold changes in hEF1 promoter activity are shown for CpG-free Lucia reporter constructs containing either methylated (red checkered) or unmethylated (blue checkered) rTHOR constructs (10). Unpaired $t$ test was used for statistical analysis ( $\left.{ }^{*} P<0.05\right)$. 

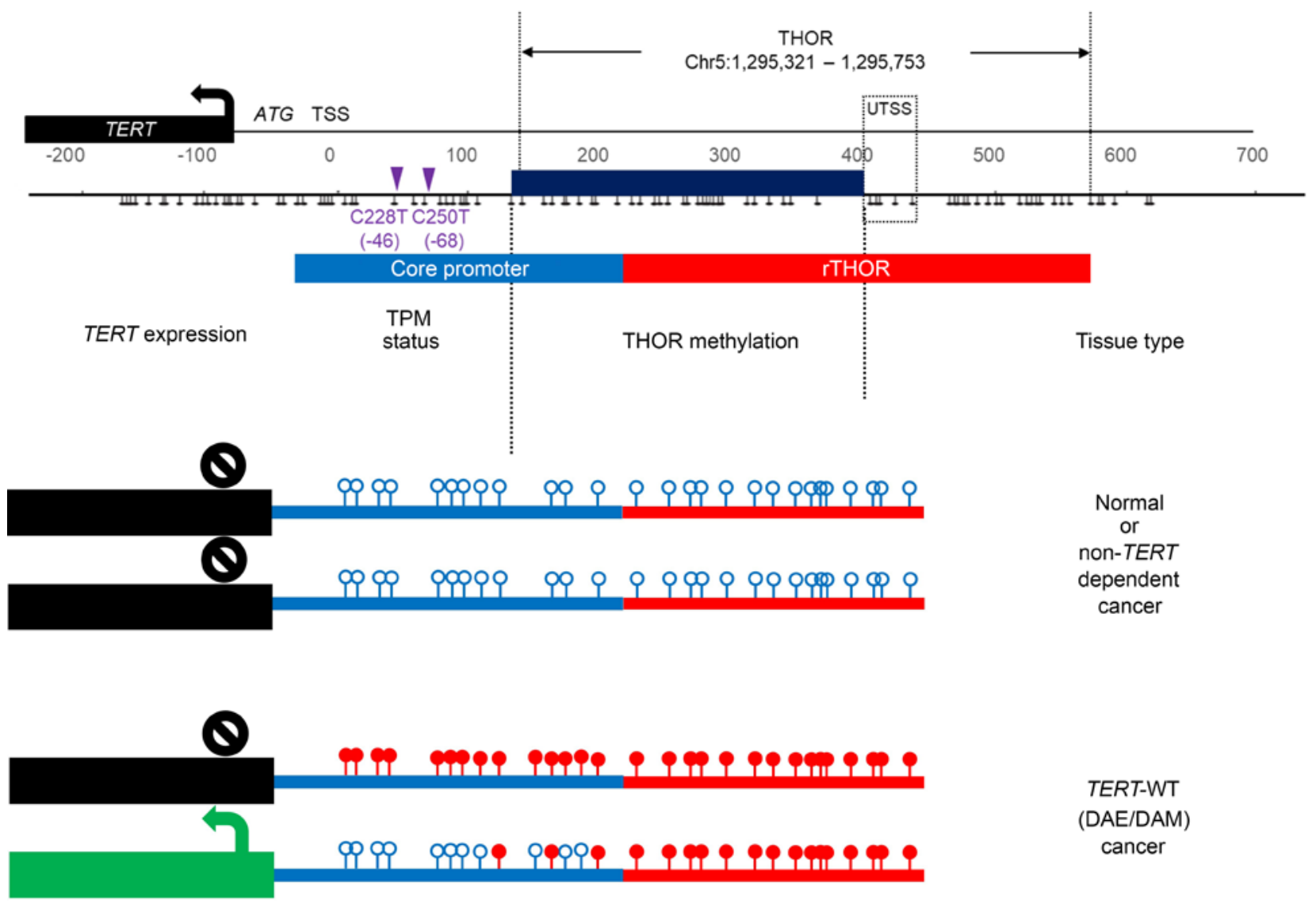

TERT-WT

(DAE/DAM)

cancer

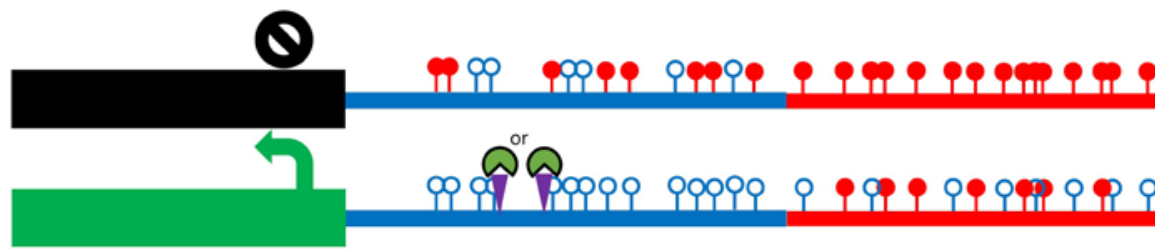

TPM

(DAE/DAM)

cancer

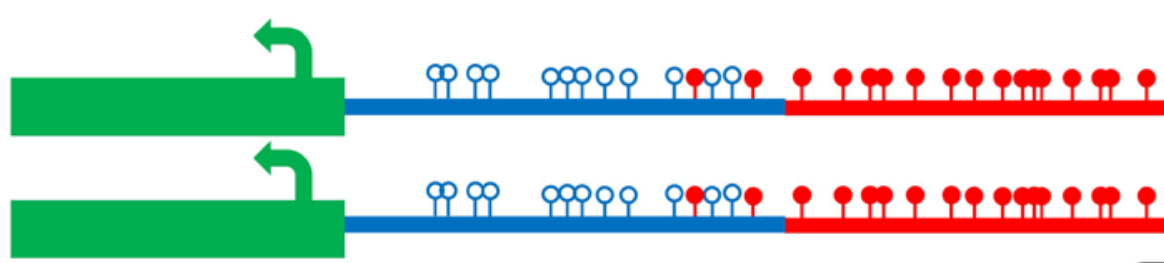

TERT-WT

(BAE/BAM)

cancer

\begin{tabular}{|c|c|c|}
\hline \multicolumn{3}{|c|}{ Legend } \\
\hline & TPM sites & ( ETS-family factor \\
\hline & Unmethylated CpG & Expression \\
\hline D & Methylated CpG & (No expression \\
\hline
\end{tabular}

Figure 5. Proposed model of epigenetic regulation of TERT in human cancer. Normal cells or non-TERT-dependent cancer cells have hypomethylated TERT promoter and no expression of TERT. In TERT promoter WT cancer cells, rTHOR is biallelically hypermethylated and the allele harboring low methylation of the core TERT promoter is expressing TERT (WT DAE). In cases of WT BAE/BAM (biallelic expressing/biallelic methylation) cancer cells, the core TERT promoter is lowly methylated in both alleles and rTHOR is biallelically hypermethylated. In TPM cancer cells, DAE of TERT is driven by an allele harboring heterozygous TPMs and low methylation of the core TERT promoter. 
ARMS-PCR. To determine which base of the promoter SNP (pSNP, rs 2853669, A or $\mathrm{G}$ ) is associated with which base of the exon SNP (exSNP, rs2736098, C or T), ARMS-PCR was utilized. The primer sets (Supplemental Table 1) target each of the 2 bases at both pSNP and exSNP. Testing all 4 possible combinations (pSNP A-exSNP C, pSNP A-exSNP T, pSNP G-exSNP C, and pSNP G-exSNP T) allows for determination of the pSNP-exSNP association in each allele present within the cell line. A separate internal primer was used to determine which allele harbored heterozygous TERT promoter mutation at C228T or C250T locus.

Relative TERT expression by digital droplet PCR. Total RNA was extracted from each tissue or cell line using Trizol reagent (Invitrogen) and $1 \mu \mathrm{g}$ total RNA was reverse transcribed to cDNA with Superscript III and IV (Invitrogen). Each cDNA sample (50 ng) was analyzed with digital droplet PCR with a probe targeting a common exon SNP (exSNP, rs2736098), and the gDNA sample was analyzed in parallel to identify allelic imbalance at the gDNA level prior to transcription. The ddPCR results were double confirmed with Sanger sequencing of the cDNA samples.

Reporter gene expression analysis. All reporter gene expression analyses include data from experimental triplicates. The significance of reporter gene expression was assessed with 1-way ANOVA with Dunnett's post hoc test for multiple comparisons. Multiple fragments of the TERT promoter (including the core TERT promoter and core + THOR) were PCR amplified and cloned into multiple cloning sites of a CpG-free reporter backbone vector (pCpGfree-promoter-Lucia, Invivogen) using FastDigest BcuI (SpeI) and NsiI (Thermo Fisher Scientific). Constructs were transformed into E. coli containing PIR1 (One Shot PIR1 Chemically Competent E. coli, Invitrogen) and amplified using Qiaprep Spin MiniPrep Kit (Qiagen). The vector DNA was then exposed to in vitro methylation using M. SssI CpG Methyltransferase (New England Biolabs Ltd.) for in vitro methylation of the inserted fragment. All constructs were transiently transfected into LN229 and HT1080 cancer cell lines. After 48 hours, light signal produced by synthetic luciferase Lucia (CpG-free synthetic luciferase) was measured using QUANTI-Luc (Invivogen) and a single-tube luminometer (Berthold Technologies).

Study approval. This study was approved by the Institutional Research Ethics Board (REB: 1000004710) at The Hospital for Sick Children (SickKids; Toronto, Ontario, Canada). Tissue samples were collected from the collaborating participating centers after approval from the local research ethics boards, including consents for the use of tissues for research purposes. Collaborating centers include the following: The Hospital for Sick Children; University Hospital Zürich; Duke University Medical Center; Toronto Western Hospital, University Health Network; Princess Margaret Cancer Centre, University Health Network; Ontario site of the Colon Cancer Family Registry (OFCCR); Ontario Institute for Cancer Research (OICR); and Medical
University of Innsbruck. See Table 1 for additional details about the collaborating centers.

Statistics. General statistical analyses were performed using a Fisher's exact test or a $\chi^{2}$ test for categorical variables, and a 2-tailed $t$ test (GraphPad Prism, version 6.0) for continuous variables. $P$ values of less than 0.05 were considered statistically significant. For functional experiment (Figure 4), 1-way ANOVA with Dunnett's post hoc test for multiple comparisons was used $\left({ }^{* * *}\right.$ adjusted $P<0.001$, ****adjusted $P<0.0001)$.

\section{Author contributions}

DDL, PCB and UT were responsible for the study concept. DDL, MK, NMN, and UT designed the experiments. DDL conducted all experiments and performed all analyses. MK performed NGS sample generation and assisted with data analysis. SS designed an in-house algorithm to analyze NGS data. CHZ assisted with cell culture and DNA extraction. JDA assisted with experiments. RL, TH, PJW, HK, FN, GZ, BHD, HY, SG, TJP, VR, and MDT provided patient samples. MK and NMN provided critical insight into writing the manuscript. DDL and UT cowrote the manuscript.

\section{Acknowledgments}

This work was supported by operating grants from the Canadian Institutes of Health Research (MOP-137899) and the Canadian Cancer Society Research Institute. Cancer samples in this study were provided by the collaborators from different centers (outlined in Table 1). Lung cancer samples included in this study were provided by the Ontario Institute for Cancer Research (OICR). Colon cancer samples included in this study were provided by the Ontario site of the Colon Cancer Family Registry (OFCCR, www.coloncfr. org). The CCFR is supported in part by funding from the National Cancer Institute (NCI) of the National Institutes of Health (NIH) (award U01 CA167551) and through cooperative agreements U01/ U24 CA074783 from the NCI (to SG). Support for case ascertainment was provided in part by the Ontario Cancer Registry. The content of this manuscript does not necessarily reflect the views or policies of the NIH or any of the collaborating centers in the CCFR, nor does mention of trade names, commercial products, or organizations imply endorsement by the US government, any cancer registry, or the CCFR. Special acknowledgement to Nicholas Fernandez for isolation of DNA from melanoma samples.

Address correspondence to: Uri Tabori, Division of Haematology/ Oncology, The Hospital for Sick Children, 555 University Avenue, Toronto, Ontario, M5G1X8 Canada. Phone: 1.416.813.7654 ext. 201503; Email: uri.tabori@sickkids.ca.
1. Jafri MA, et al. Roles of telomeres and telomerase in cancer, and advances in telomerase-targeted therapies. Genome Med. 2016;8(1):69.

2. d'Adda di Fagagna F, et al. A DNA damage checkpoint response in telomere-initiated senescence. Nature. 2003;426(6963):194-198.

3. Harley CB, et al. Telomeres shorten during ageing of human fibroblasts. Nature. 1990;345(6274):458-460.

4. Gunes C, Rudolph KL. The role of telomeres in stem cells and cancer. Cell. 2013;152(3):390-393.
5. Finkel T, et al. The common biology of cancer and ageing. Nature. 2007;448(7155):767-774.

6. Kyo S, et al. Understanding and exploiting hTERT promoter regulation for diagnosis and treatment of human cancers. Cancer Sci. 2008;99(8):1528-1538.

7. Gay-Bellile M, et al. TERT promoter status and gene copy number gains: effect on TERT expression and association with prognosis in breast cancer. Oncotarget. 2017;8(44):77540-77551.

8. Horn S, et al. TERT promoter mutations in familial and sporadic melanoma. Science.
2013;339(6122):959-961.

9. Huang FW, et al. Highly recurrent TERT promoter mutations in human melanoma. Science. 2013;339(6122):957-959.

10. Lee DD, et al. DNA hypermethylation within TERT promoter upregulates TERT expression in cancer. J Clin Invest. 2019;129(1):223-229.

11. Esopi D, et al. Pervasive promoter hypermethylation of silenced TERT alleles in human cancers Cell Oncol (Dordr). 2020;43(5):847-861.

12. Huang FW, et al. TERT promoter mutations and 
monoallelic activation of TERT in cancer. Oncogenesis. 2015;4:e176.

13. Stern JL, et al. Allele-specific DNA methylation and its interplay with repressive histone marks at promoter-mutant TERT genes. Cell Rep. 2017;21(13):3700-3707.

14. Stern JL, et al. Mutation of the TERT promoter, switch to active chromatin, and monoallelic TERT expression in multiple cancers. Genes Dev. 2015;29(21):2219-2224.

15. Walker EJ, et al. Monoallelic expression determines oncogenic progression and outcome in benign and malignant brain tumors. Cancer Res. 2012;72(3):636-644.

16. Reuveni E, et al. Differential contribution of cis and trans gene transcription regulatory mechanisms in amygdala and prefrontal cortex and modulation by social stress. Sci Rep. 2018;8(1):6339.

17. Baylin SB. DNA methylation and gene silencing in cancer. Nat Clin Pract Oncol. 2005;2(suppl 1):S4-S11.

18. Clark SJ, Melki J. DNA methylation and gene silencing in cancer: which is the guilty party? Oncogene. 2002;21(35):5380-5387.

19. Castelo-Branco P, et al. A cancer specific hyper- methylation signature of the TERT promoter predicts biochemical relapse in prostate cancer: a retrospective cohort study. Oncotarget. 2016;7(36):57726-57736.

20. Leao R, et al. Combined genetic and epigenetic alterations of the TERT promoter affect clinical and biological behavior of bladder cancer. Int $J$ Cancer. 2019;144(7):1676-1684.

21. Newton CR, et al. Analysis of any point mutation in DNA. The amplification refractory mutation system (ARMS). Nucleic Acids Res. 1989;17(7):2503-2516.

22. Fan Y, et al. Telomerase expression by aberrant methylation of the TERT promoter in melanoma arising in giant congenital nevi. J Invest Dermatol. 2016;136(1):339-342.

23. Seynnaeve B, et al. Genetic and epigenetic alterations of TERT are associated with inferior outcome in adolescent and young adult patients with melanoma. Sci Rep. 2017;7:45704.

24. Wu Y, et al. Telomerase reverse transcriptase methylation predicts lymph node metastasis and prognosis in patients with gastric cancer. Onco Targets Ther. 2016;9:279-286.

25. Zhang H, et al. Promoter hypermethylation of
TERT is associated with hepatocellular carcinoma in the Han Chinese population. Clin Res Hepatol Gastroenterol. 2015;39(5):600-609.

26. Amirian ES, et al. Predictors of survival among pediatric and adult ependymoma cases: a study using surveillance, epidemiology, and end results data from 1973 to 2007. Neuroepidemiology. 2012;39(2):116-124.

27. Brawer MK. Prostatic intraepithelial neoplasia: an overview. Rev Urol. 2005;7 Suppl 3:S11-S18.

28. Cesare AJ, Reddel RR. Alternative lengthening of telomeres: models, mechanisms and implications. Nat Rev Genet. 2010;11(5):319-330.

29. Castelo-Branco P, et al. Methylation of the TERT promoter and risk stratification of childhood brain tumours: an integrative genomic and molecular study. Lancet Oncol. 2013;14(6):534-542.

30. Rowland TJ, et al. Allele-specific proximal promoter hypomethylation of the telomerase reverse transcriptase gene (TERT) associates with TERT expression in multiple cancers. Mol Oncol.2020;14(10):2358-2374.

31. Ramlee MK. et al. Transcription regulation of the human telomerase reverse transcriptase (hTERT) gene. Genes (Basel). 2016;7(8):50. 